$28^{\text {th }}$ AIAA Applied Aerodynamics Conference - CFD Drag Prediction Workshop Results, 28 June -1 July 2010, Chicago IL

\title{
Application of Novel Hybrid Mesh Generation Methodologies for Improved Unstructured CFD Simulations
}

\author{
Simone Crippa*
}

\begin{abstract}
On the basis of the DLR contribution to DPW4, a procedure to produce an unstructured grid-convergence family as self-similar as possible is documented, with a specific procedure for steering the near-field, advancing-layer process. A novel hybrid mesh generation approach is presented, in which adjoint-based dissipation error evaluations are successfully employed to improve the solution accuracy for specific conditions. An intrinsic deficit of the standard mesh generation procedure, results in an unsatisfying resolution of flow features at the wing-body junction. For this case, an alternative solution strategy based on chimera grids is tested and found to improve the aerodynamic evaluation.
\end{abstract}

\section{Introduction}

The successful drag prediction workshop series set the focus of it's fourth gathering (DPW4) in the "blind" prediction of drag and moment coefficients of the NASA common research model ${ }^{1}(\mathrm{CRM})$ transonic wing-body-tail configuration. One of the main objectives of DPW4 is to evaluate the performance of stateof-the-art Navier-Stokes codes, thus this study documents some of the steps undertaken at the Institute of Aerodynamics and Flow Technology of DLR, to prepare the contribution to the DPW4. To identify possible CFD areas needing additional research and development, both standard procedures were used in this study and advanced methodologies, such as new grid generation methods and advanced turbulence models.

The CFD solver employed for this study, the DLR TAU code,${ }^{2}$ is used here in conjunction with the most widely used one- and two-equation turbulence models (Spalart-Allmaras ${ }^{3}$ and Menter SST k- $\omega^{4}$ ), but also with a differential Reynolds stress models (SSG/LLR- $\left.\omega^{5}\right)$.

The hybrid, quad-dominant grid generation package Solar ${ }^{6}$ is mainly used in the semi-automated mode. Hereby the geometry of the configuration at hand is subdivided in several zones of either lifting surfaces or bodies. Afterwards a set of volume sources are automatically distributed for each component according to so-called philosophy files in which accumulated knowledge and best-practice guidelines are collected. For the purposes of the DPW4 contribution, these sources are then modified and manually complemented in certain regions of interest.

The DPW committee releases a set of guidelines to guarantee similar grid quality throughout all workshop contributions. In some points the guidelines discern between structured and unstructured grids. This is necessary, as the generation of a family of truly self-similar unstructured grids is not possible with the standard advancing-layer/advancing-front approaches. Here one of the key design features of Solar can be used to achieve a high level of self-similarity between the grid levels. Only one set of volumetric, background sources are used to steer the complete mesh generation process. Thus by scaling appropriately the target spacing of these sources an influence on the complete grid (surface and volume) is achieved.

The complete DPW4 dataset produced by DLR is the basis for a detailed aerodynamic assessment by Brodersen et al. ${ }^{7}$ A subset of the data is used here to elucidate on the pre-workshop grid generation procedure and a post-workshop discretization improvement effort.

\footnotetext{
${ }^{*}$ Research associate, DLR Institute of Aerodynamics and Flow Technology, $\mathrm{C}^{2} \mathrm{~A}^{2} \mathrm{~S}^{2} \mathrm{E}$, Lilienthalplatz 7, 38108 Braunschweig, Germany, +49 531 2953610; AIAA Member.

Copyright (C) 2010 by Simone Crippa. Published by the American Institute of Aeronautics and Astronautics, Inc. with permission.
} 


\section{Cases and Grids Description}

During the initial grid evaluation process, it became clear that the sharp ending of the body tail cone onto the symmetry plane is detrimental to the convergence of the available Reynolds stress models. The precise reason for this is not understood yet, but a workaround is found by slightly modifying the tail cone trailing edge. The sharp trailing edge is cut off at $\mathrm{x}=2561.5 \mathrm{inch}$, resulting in a blunt trailing edge of 0.5 inch thickness. For the sake of consistency, the modified geometry is used for all cases/computations.

For none of the grids, point 2)h)ii) of the gridding guidelines (version number 4, November $3^{\text {rd }} 2008$ ) is fulfilled. The requirement sets the wing and tail span-wise grid spacing at root at approx. $0.1 \%$ local semispan. Due to the advancing layer approach used in Solar, in concave corners the wall-normal extrusion of the surface mesh may result in layer intersections. To avoid these intersections the expansion ratio is locally reduced, which as a result leads to local contraction of the prismatic/hexahedral layer. If the span-wise spacing at the wing and tail root is too small, the contraction of the layer may be so extreme, that the boundary layer edge in the concave corner is not resolved with prisms/hexahedra. This results in a lower limit for the span-wise spacing that is higher than the value required by the gridding guidelines. For the medium grid, the span-wise spacing at the root is increased to approx. $0.8 \%$ local semi-span.

The full contribution of DLR to DPW4 covers three of the four cases defined by the DPW committee, case 1.1, 1.2 and 3 , requiring in total seven separate grids.

\section{II.A. Case 1.1}

Case 1.1 refers to the grid convergence study, performed on the wing-body-tail CRM configuration with the tail deflection angle of $0^{\circ}$. At least three solutions on a family of grids of varying size are required to perform Richardson extrapolation. The complete grid family has to lie within the asymptotic grid convergence range, this implies that all relevant aerodynamic phenomena have to be resolved on all three levels. For the three required grids (coarse, medium and fine) the guidelines call for a ratio of three in total number of points between the grid levels. Due to the requirement in Solar to specify the target grid spacing in distinct volume sources, a one-dimensional refinement/coarsening factor is required. A factor of three for a three-dimensional grid translates to a one-dimensional scaling factor of $\sqrt[3]{3}$, approx. 1.4422. Only this factor is used to scale the volumetric sources, whereas the range of influence of the sources is not changed as it is linked to the geometry. A further requirement of the gridding guidelines sets the minimal resolution across the thick trailing edges of the lifting surfaces. To comply with this requirement, the three grids prepared for the workshop feature 8,12 and 18 cells across the trailing edges. A summary of the final grid family points and elements counts is given in table 1 .

Table 1. Overall grid details for case 1.1.

\begin{tabular}{llll}
\hline Grid & coarse & medium & fine \\
\hline \hline Surface points & $130 \cdot 10^{3}$ & $271 \cdot 10^{3}$ & $566 \cdot 10^{3}$ \\
No. of points in prism/hexa layer & $3.47 \cdot 10^{6}$ & $9.94 \cdot 10^{6}$ & $28.69 \cdot 10^{6}$ \\
No. of tetrahedral cells & $5.31 \cdot 10^{6}$ & $14.31 \cdot 10^{6}$ & $38.58 \cdot 10^{6}$ \\
Total no. of points & $4.07 \cdot 10^{6}$ & $11.70 \cdot 10^{6}$ & $34.08 \cdot 10^{6}$ \\
Total grid size ratio (medium grid as reference) & $1 / 2.87$ & 1 & 2.91 \\
Total no. of cells & $8.56 \cdot 10^{3}$ & $23.78 \cdot 10^{6}$ & $66.65 \cdot 10^{6}$ \\
\hline
\end{tabular}

\section{II.A.1. Near-Field Grid Generation Procedure}

The input values required by the advancing layer grid generation process, first layer spacing and expansion ratio, have to be chosen wisely. During the grid generation and evaluation process for DPW4, it was discovered that a similar total layer thickness between the grid levels is of paramount importance for a successful, unstructured grid-convergence study. It is important to resolve the same physical region on the various grid levels with the same element types. A similar near-field extent normal to the walls, guarantees that the transition location from hexahedral/prismatic elements to tetrahedra is similar between the grid levels. Having the element type transitions in the same physical region allows to capture in a self-similar 
way, on all grid levels, eventual discretization errors. The relations between the grid levels in terms of first layer spacing and number of wall-normal layers should follow the scaling factor given above.

Given the requirement for a self-similar total layer thickness, scaled first layer spacing and scaled total amount of layers, leaves only the expansion ratio as variable to be determined. The geometric series for the total layer thickness $(H)$ is

$$
\begin{aligned}
H & =\sum_{i=0}^{n} a \cdot q^{i} \\
& =a \cdot \frac{1-q^{n+1}}{1-q}
\end{aligned}
$$

where the total number of layers is $N=n+1$, the expansion ratio is $q$ and the first layer spacing is $a$. Keeping the total layer thickness between two grid levels constant $\left(H_{1}=H_{2}\right)$, results in

$$
a_{1} \cdot \frac{1-q_{1}^{N_{1}}}{1-q_{1}}=a_{2} \cdot \frac{1-q_{2}^{N_{2}}}{1-q_{2}} .
$$

Hereby the relation between $a_{1}$ and $a_{2}$, as well as $N_{1}$ and $N_{2}$, is set by the scaling factor $\sqrt[3]{3}$; for example, with grid level 2 being finer than grid level 1 , follows $a_{2}=a_{1} / \sqrt[3]{3}$ and $N_{2}=N_{1} \cdot \sqrt[3]{3}$. Starting with a sensible value for the expansion ratio $q_{1}$ and a total amount of layers $N_{1}$, the only unknown in equation (1) is $q_{2}$, which can be computed iteratively.

For the DPW4 grid-convergence family, the values for the coarse and fine levels are derived from the medium grid. The first layer spacings given in the gridding guidelines are used, as the scaling factor of 1.5 is sufficiently close to $\sqrt[3]{3}$. The resulting values for the near-field mesh are summarized in table 2 . Note that

Table 2. Near-field grid details.

\begin{tabular}{llll}
\hline Grid & coarse & medium & fine \\
\hline \hline First wall-normal layer spacing $(a)$ & $1.478 \cdot 10^{-3}$ inch & $9.85 \cdot 10^{-4}$ inch & $6.57 \cdot 10^{-4}$ inch \\
No. of cells with constant spacing & 3 & 3 & 3 \\
Expansion ratio $(q)$ & 1.3009 & 1.2 & 1.135 \\
Max. no. of wall-normal layers $(N)$ & 30 & 42 & 60 \\
\hline
\end{tabular}

for full consistency, the number of wall-normal layers with constant spacing should also be scaled by $\sqrt[3]{3}$, but neglecting this was not deemed of primary influence to the results. Furthermore, note that the expansion ratios of table 2 fulfill the requirement given in the gridding guidelines only for the medium and fine grids. The expansion ratio of the coarse grid is larger than the defined, maximal value of 1.25.

A comparison of the three final grids is shown in figure 1, where the self-similar relation between the three levels is visible in the highlighted region. In wall-tangential direction, the factor of approx. 1.5 can be recognized by the cascade of 2, 3 and 4.5 quadrilateral elements. A similar wall-normal total layer extent is also recognizable.

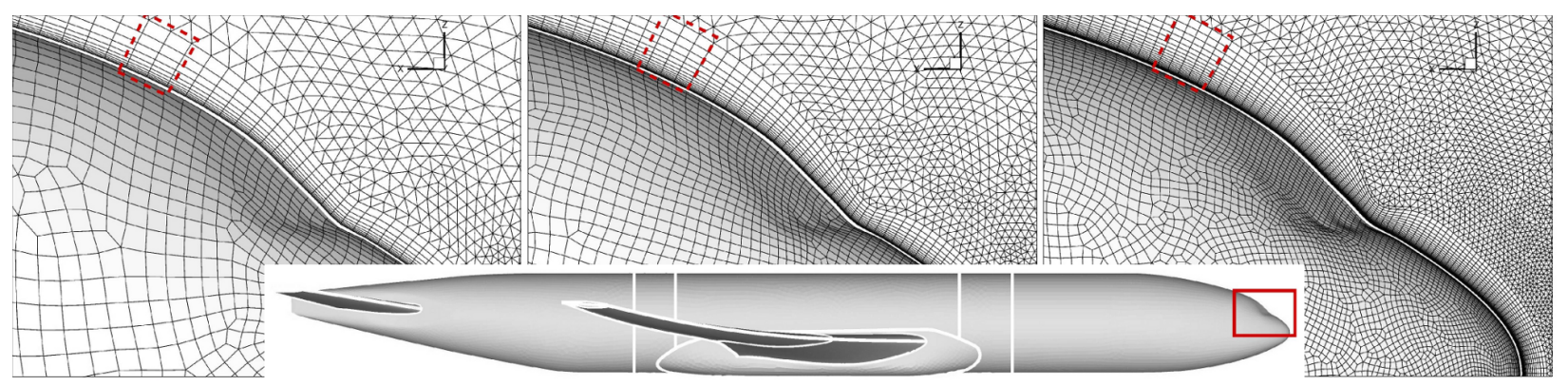

Figure 1. Comparison of coarse, medium and fine grids (case 1.1); lateral view on fore-body with symmetry. 


\section{II.B. $\quad$ Case 1.2}

For case 1.2, that refers to a trim drag analysis by variation of the tail deflection angle, the same sources as for the medium grid of case 1.1 are used. The sources of the tail geometry are rotated according to the geometric tail deflection (WBT ih $=-2^{\circ}, 0^{\circ}, 2^{\circ}$ ) or neglected altogether for the configuration without tailplane (WB). The resulting grid sizes are summarized in table 3. For the scope of this study, only a subset of the case 1.2 data is used, namely the data at the angle of attack of $4^{\circ}$.

Table 3. Overall grid details for case 1.2

\begin{tabular}{lllll}
\hline Grid & WB & WBT ih- $2^{\circ}$ & WBT ih0 & WBT ih $+2^{\circ}$ \\
\hline \hline Surface points & $208 \cdot 10^{3}$ & $271 \cdot 10^{3}$ & $271 \cdot 10^{3}$ & $271 \cdot 10^{3}$ \\
Max. no. of wall-normal layers & 43 & 42 & 42 & 42 \\
No. of points in prism/hexa layer & $7.32 \cdot 10^{6}$ & $9.94 \cdot 10^{6}$ & $9.94 \cdot 10^{6}$ & $9.94 \cdot 10^{6}$ \\
No. of tetrahedral cells & $4.62 \cdot 10^{6}$ & $12.76 \cdot 10^{6}$ & $14.31 \cdot 10^{6}$ & $14.65 \cdot 10^{6}$ \\
Total no. of points & $8.59 \cdot 10^{6}$ & $11.45 \cdot 10^{6}$ & $11.70 \cdot 10^{6}$ & $11.71 \cdot 10^{6}$ \\
Total no. of cells & $17.29 \cdot 10^{3}$ & $22.24 \cdot 10^{6}$ & $23.78 \cdot 10^{6}$ & $24.1 \cdot 10^{6}$ \\
\hline
\end{tabular}

\section{II.C. Case 3}

Case 3 is required to assess the Reynolds number scaling effects, by generating a mesh similar to the medium mesh of case 1.1, but with modified first layer spacing for a four times higher Reynolds number. The same surface mesh as for the medium grid is used, but the first layer spacing is set to 0.000273 inch. This results in the extrusion of max. 49 layers, instead of 42 as for case 1.1, with a similar total layer extension. The total number of points for this case is $13.24 \cdot 10^{6}$, with $11.53 \cdot 10^{6}$ points or $11.08 \cdot 10^{6}$ elements in the near-field region, and $14.01 \cdot 10^{6}$ tetrahedra. Results from case 3 are not presented in this study.

\section{Detailed Grid Analysis and Improvement}

Using the Solar standard procedures, an initial grid family for case 1.1 was generated. For the initial grid family, the main difference from the standard procedure consists in adding a chord-wise refinement at the wing mid-chord over the entire span. The initial grid family was then checked for convergence behavior, and although satisfactory at first sight, an improvement was sought.

The error due to dissipation for the medium grid in respect to two cost functions (drag and moment coefficients) is evaluated using the discrete adjoint solver available in the TAU code. ${ }^{8}$ The adjoint solver does not only output the total error estimate of the evaluated cost function, but also the scaled contribution of each cell. The local error variable scaled by the cell volume (V4) is used to assess if the initial source distribution and spacing is appropriate throughout the field. By visualizing the cells with a local error variable higher than a reasonable threshold, several regions can be identified that have a major contribution to the total error, see figure 2.

Due to the a-priori refinement at mid-chord, the adjoint dissipation error assessment shows that this region is indeed sufficiently refined. Through careful, interactive visualization, several problematic regions can be identified that were not known a-priori to play a major role. Some of the regions are

- upstream of the entire wing;

- just upstream of the junction of body and wing leading edge;

- well above the model, approximately at the location of the wing-body junction;

- between the wing and the tailplane;

- upper side of the wing-body intersection between wing root mid-chord and trailing edge;

- upper and lower tailplane-body junction from leading to trailing edge; and

- below the aft-body cone. 

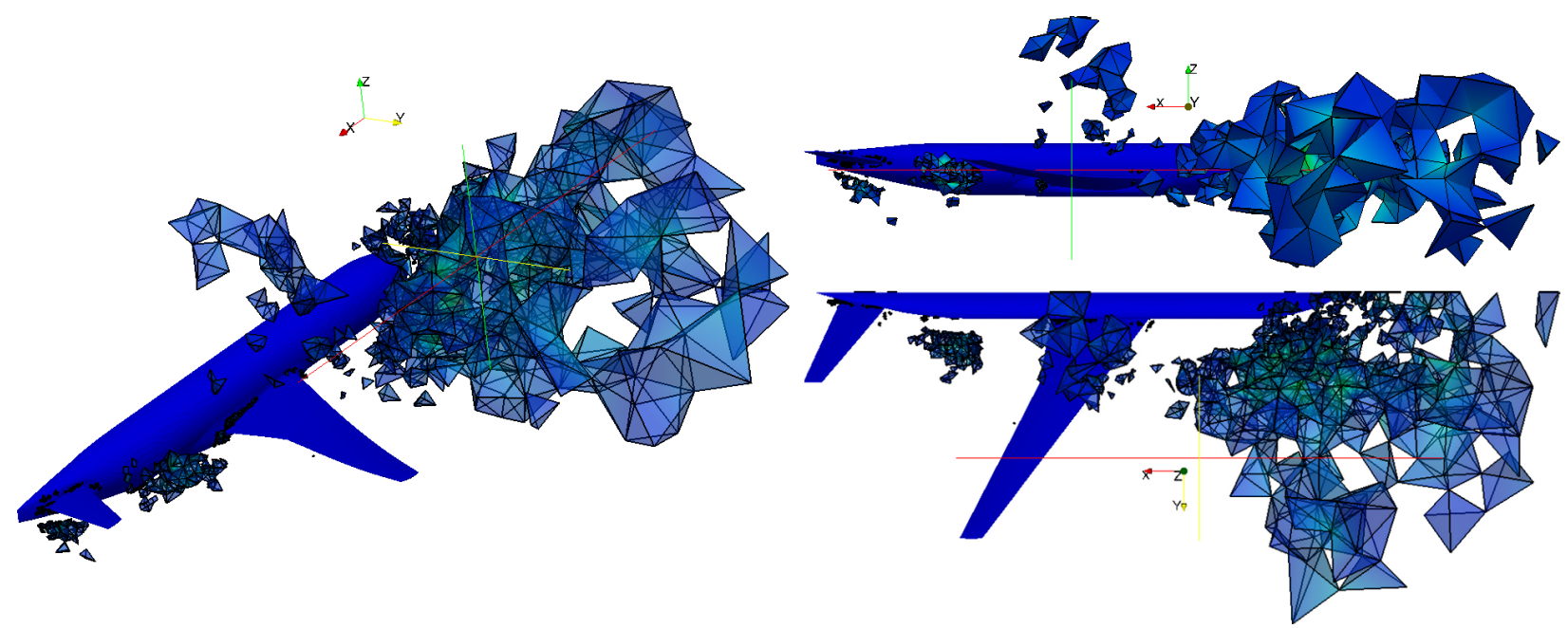

Figure 2. Case 1.1, medium grid; cells with a local error of drag coefficient larger than $5 \cdot 10^{-7}$; isometric/upstream, lateral (y-) and top (z-) views.

The field distribution of the discretization error variable for the moment coefficient shows similar problematic regions as for the drag coefficient, although with higher absolute values of the error. This is consistent with the conjectured higher sensitivity to discretization errors of the moment coefficient compared to the drag coefficient. A significant topological difference between the two evaluations is only found in respect to the surface discretization and the corresponding first few wall-normal cells.
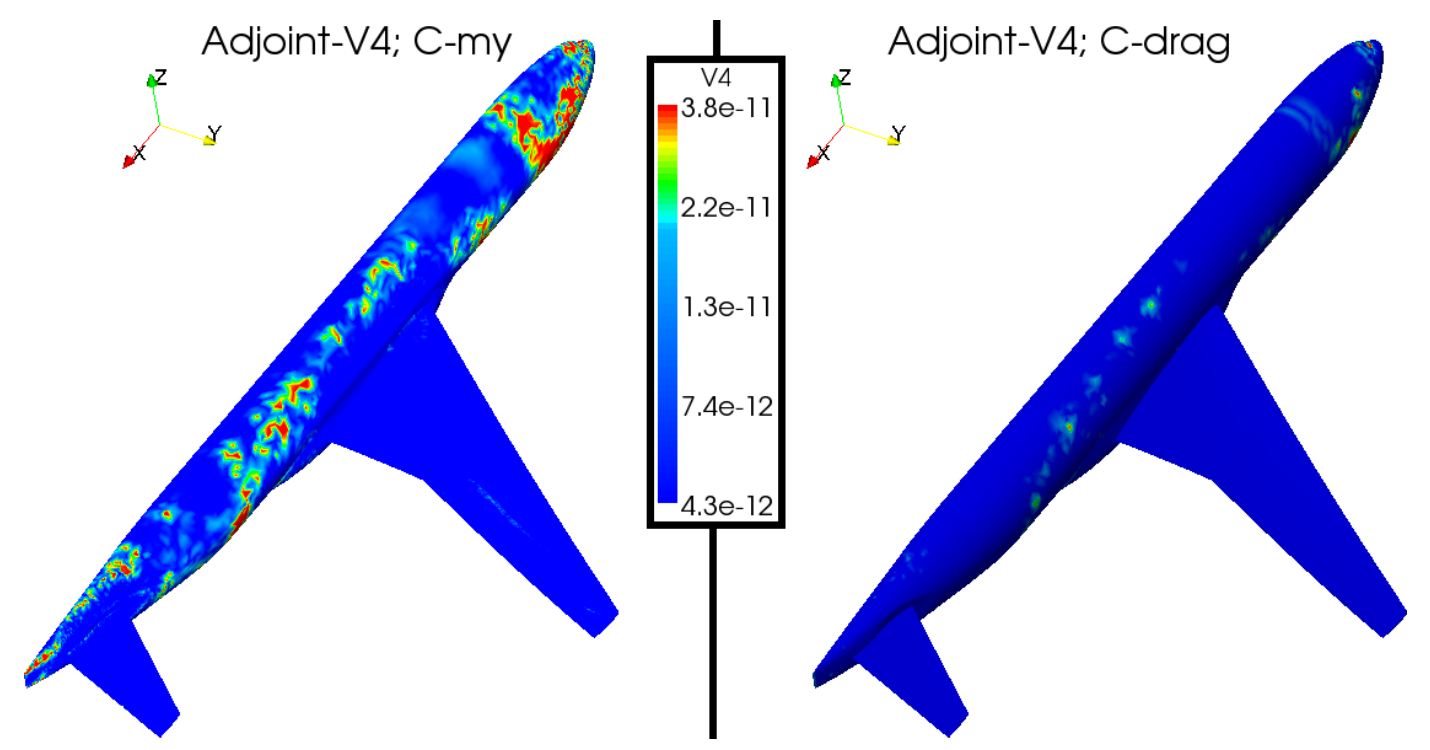

Figure 3. Case 1.1, medium grid; local dissipation error of pitch moment (C-my) and drag (C-drag) coefficients on surface elements; isometric/upstream view.

Figure 3 shows the relative sensitivity to the same surface discretization for the two cost functions. It is clear that the moment coefficient adjoint solution identifies a main contribution from the fore-body. This is understandable as the front part of the body contributes through a longer moment arm to the integrated value of the moment coefficient. Thus a small error in this region has a larger effect on the moment coefficient than on the drag coefficient.

The detailed grid analysis as mentioned above, is used to manually adapt and complement the sources for the final family of grids. Through the adjoint-based field analysis, it is also possible to identify regions where the cell spacing is "too small". Thus it is possible to perform a trade-off evaluation in terms of dissipation, and thus grid discretization error, and limit the increase of total grid points to approx. ten percent for the 
medium grid.

The grid convergence for the two grid families (initial and final) is shown in figure 4, whereby the grid improvement from the adjoint-based analysis is clearer for the drag coefficient.

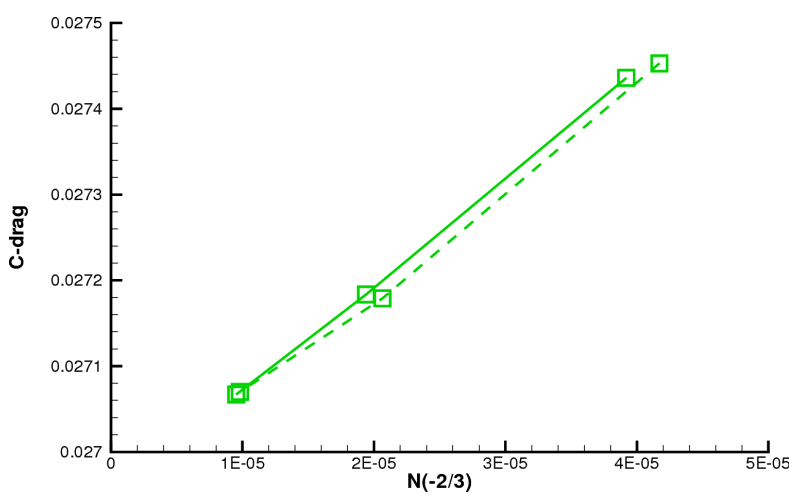

(a) Drag coefficient (C-drag)

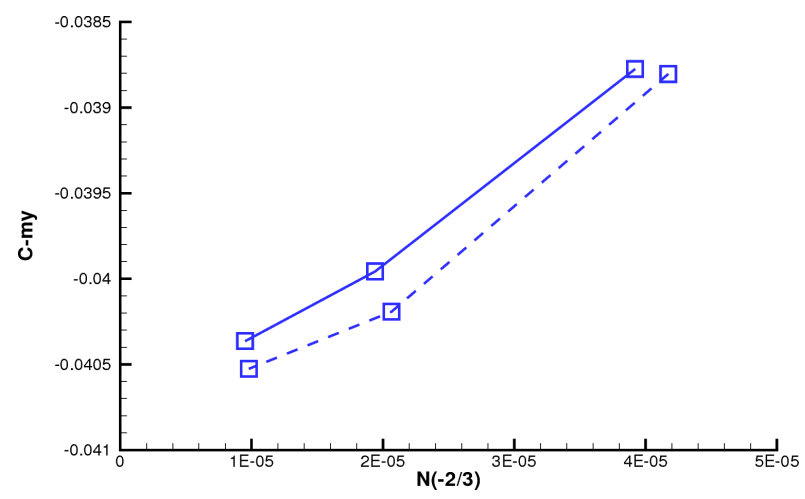

(b) Pitch moment coefficient (C-my)

Figure 4. Case 1.1, grid convergence for the initial grid family (- - ) and the adjoint-based improved family (-).

\section{Discretization Improvement through Chimera Technique}

For the DPW4 held in San Antonio, TX, on June 20-21 2008, a majority of contributions detected small separated regions at the trailing edge of the wing and tailplane junctions with the body. The existence or absence of the separation bubbles was found to be neither coupled to a solver type (unstructured or structured) nor a specific physical modelling approach. The results on the Solar grids from two participants, using in total four different turbulence models, do not feature these small separations.

An insight gained through the adjoint dissipation error evaluation, is that the wing-body and tailplanebody junction regions are not discretized sufficiently well, see figure 2 . The span-wise field cut at $\mathrm{x}=1400$ inch (wing-body) reveals that the contracted near-field mesh leads to very large tetrahedra in the concave corners that are not adequate to resolve the edge of the boundary layer, see figure 5. A way to alleviate this problem

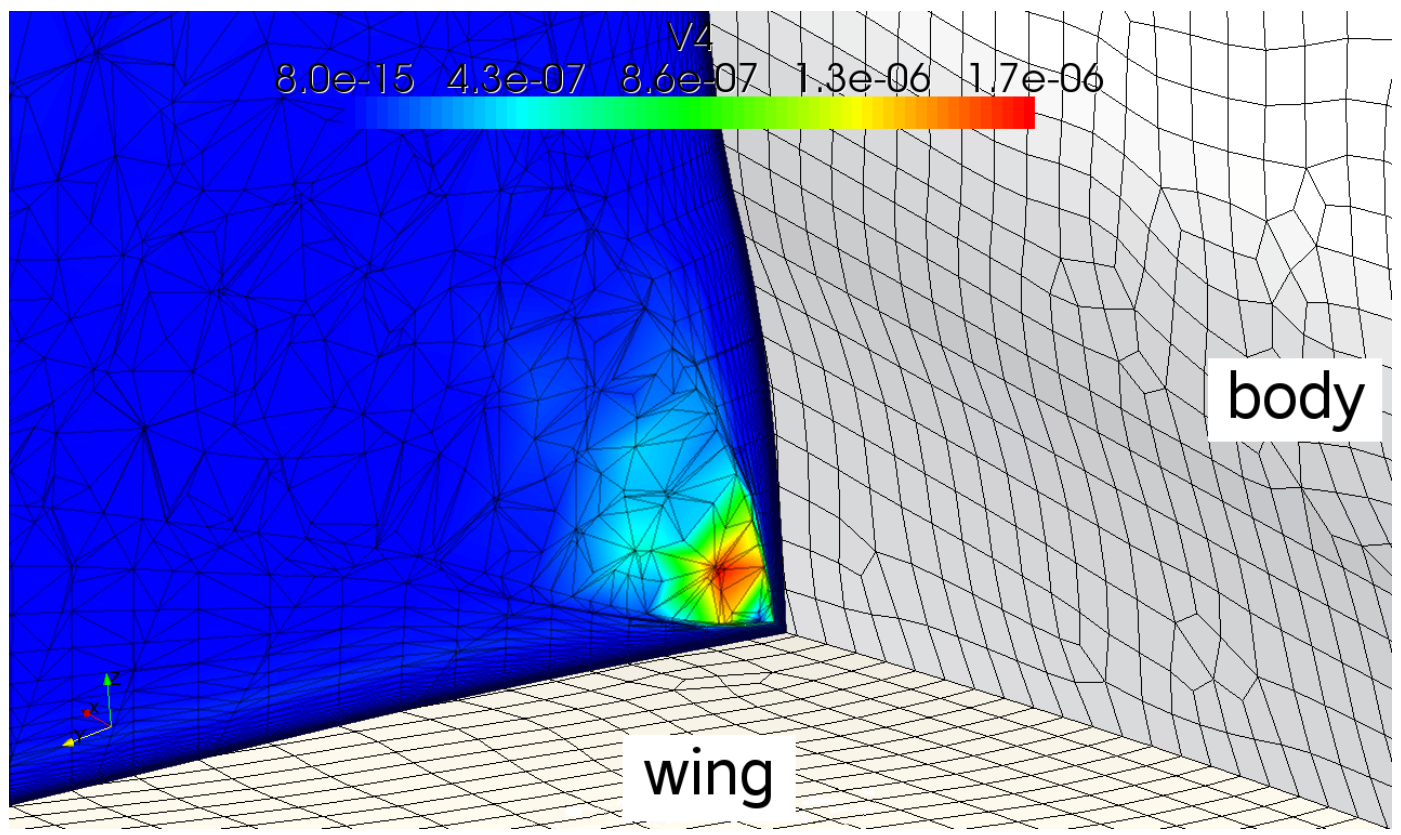

Figure 5. Case 1.1, medium grid; local dissipation error of drag coefficient on field cut-plane at $\mathrm{x}=1400$ inch; isometric/downstream view.

is described in section II, but the procedure is not enough to fully resolve the problem. A simple solution to 
this problem is not known, thus this problematic region was not fully fixed for the contribution to DPW4. The near-field layer contraction in these concave regions can not be completely excluded from the Solar grid generation process, thus a solution is sought on the solver side.

Since recently,${ }^{9}$ the TAU code has the capability to compute on chimera (overset) grids with overlapping viscous boundaries. To make use of this capability, a fully hexahedral grid was generated with a C-H topology around the complete wing airfoil and some of the wake at the wing root, see figure 6 .
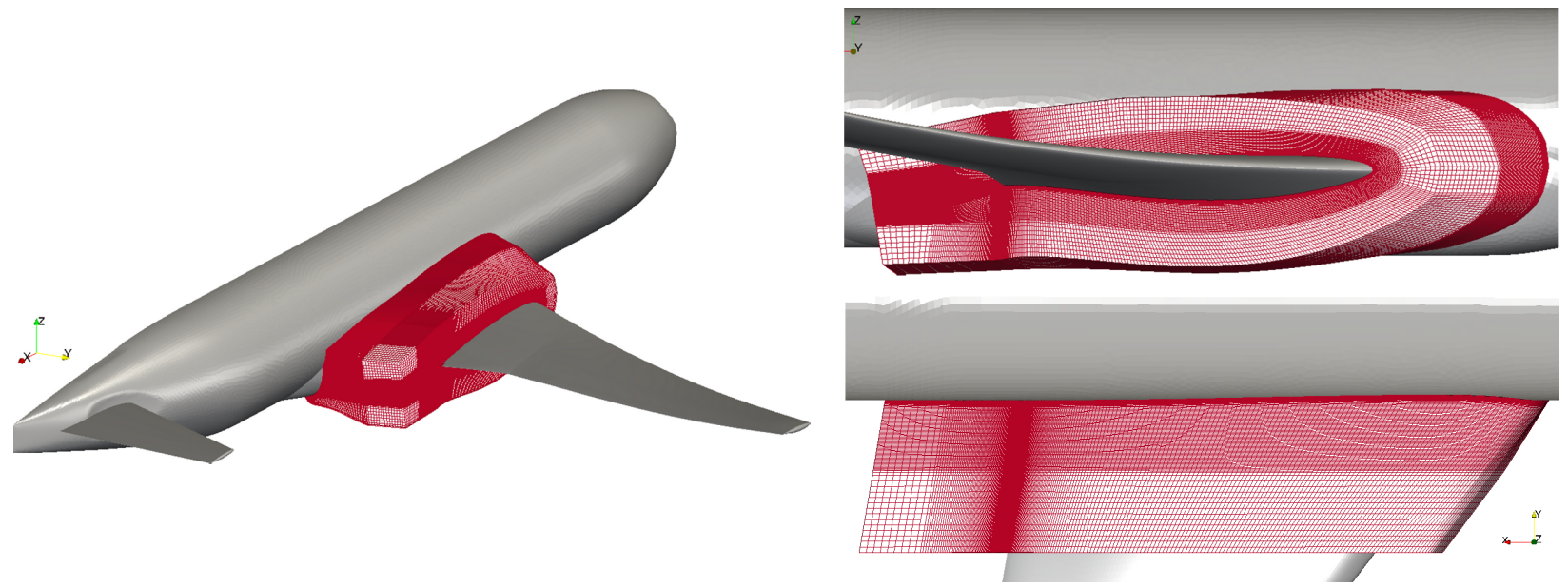

Figure 6. Additional hexahedral block; isometric/upstream, lateral and top view.

The grid spacing in the overlap/interpolation region is similar to the medium Solar grid, but the resolution at the wing-body junction is improved due to the chosen H-topology, as opposed to an O-grid topology. The additional hexahedral grid features 5.251 .719 points, 5.140 .736 elements with 391 points on the upper and lower wing airfoil, 74 points normal to the wing, 129 points normal to the body and 13 points (12 elements) across the blunt trailing edge. Hereafter, the chimera grid composed of the case 1.1 medium grid and the approx. five million elements, hexahedral grid is referred to as "SolarChimera5", whereas the initial medium grid plainly "Solar". A comparison between the Solar and SolarChimera5 grids at the wing-body junction is shown in figure 7.

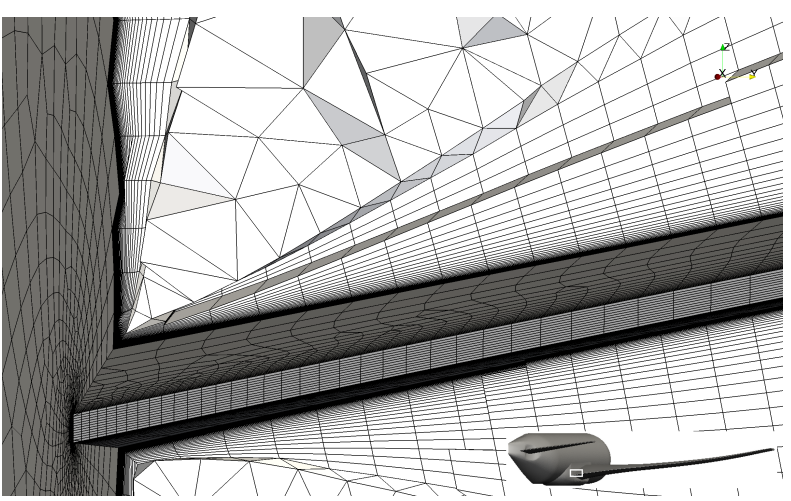

(a) Solar grid

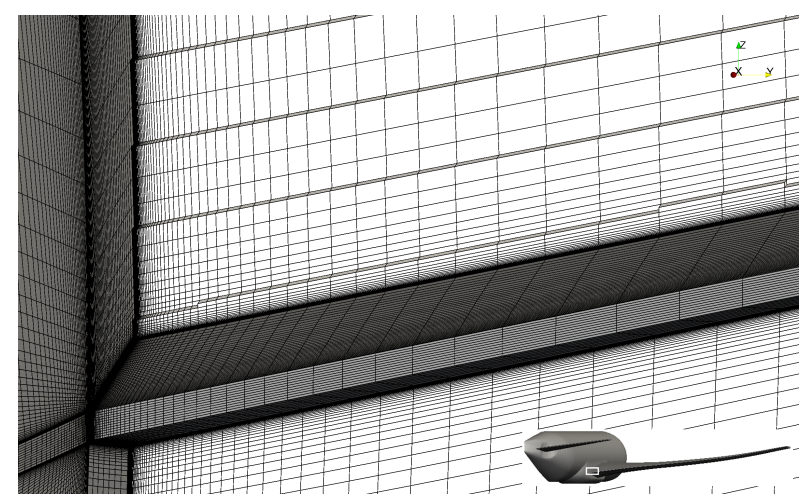

(b) SolarChimera5 grid

Figure 7. Comparison of SolarChimera5 and Solar medium grid at $\mathrm{x}=1454$ inch plane; viscous wall surface in dark grey, field cut in white.

\section{IV.A. Case 1.1 - Target Lift Coefficient of 0.5}

The solution with Spalart-Allmaras on the chimera grid, using the same far-field settings as the plain Solar grid, reveals a separation pattern similar to those observed by other DPW4 participants, see figure 8. From the result on the SolarChimera5 grid, the center of the separation bubble on the upper wing surface is found approximately at $\mathrm{x}=1454$ inch, $\mathrm{y}=124$ inch. 


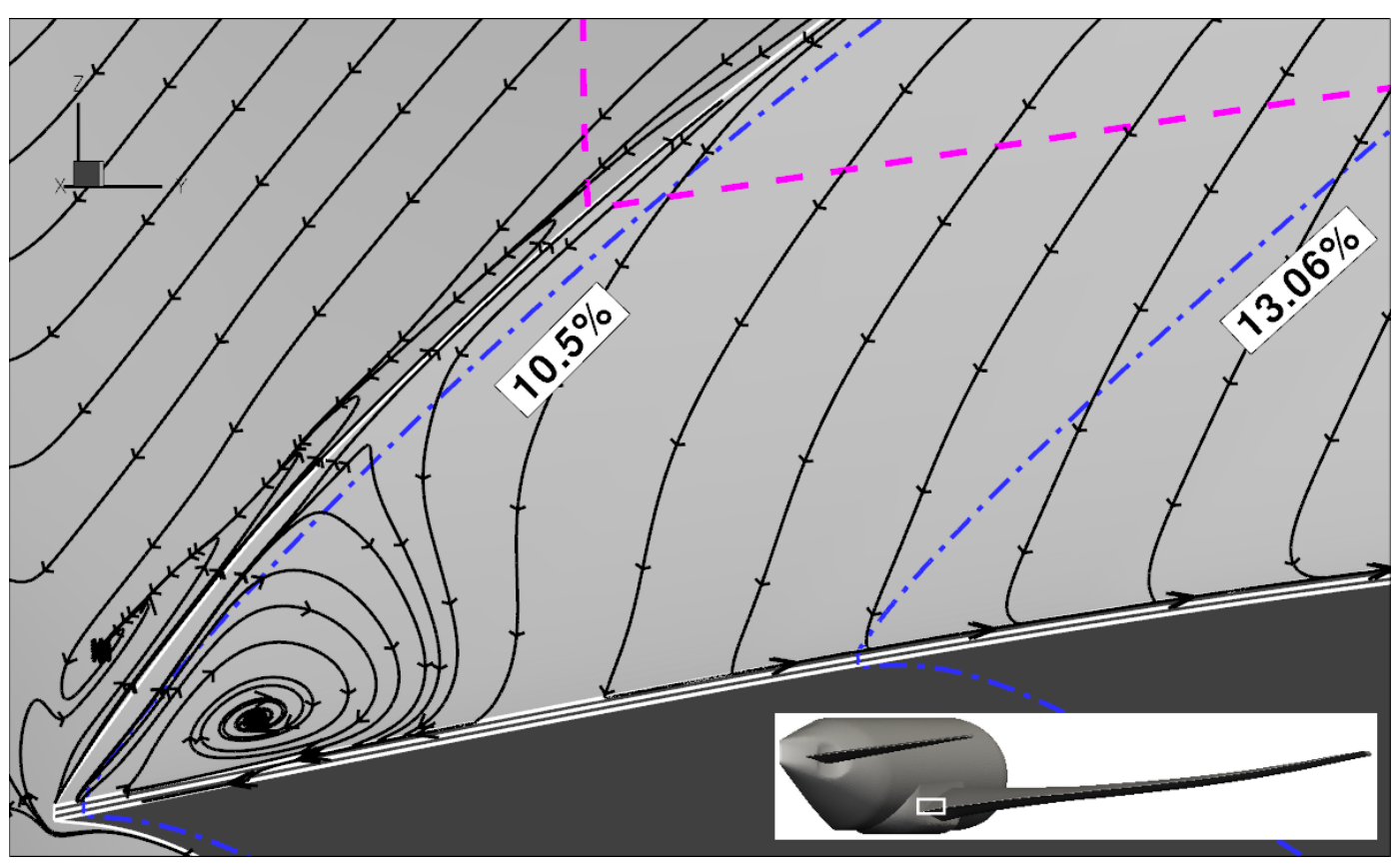

Figure 8. Skin-friction lines, $\mathbf{c}_{p, \text { crit }}(---)$ and selected surface cut-planes (- - ); SolarChimera5 grid.

By plotting the critical pressure coefficient $\left(\mathrm{c}_{p, c r i t}\right)$ as in figure 8 , it is possible to identify the sonic line impinging on the corner boundary layer. The sonic line is found at $x / c_{\text {root }} \approx 0.74$, with a wing root chord $\left(c_{\text {root }}\right)$ of 466 inch. The separation bubble in the junction starts upstream of the shock at $x / c_{\text {root }} \approx 0.68$, and remains at first constrained to a small region in the corner up until $x / c_{\text {root }} \approx 0.86$, where it expands substantially.

Due to the small extent of the separation bubble, the first available experimental pressure tap row at the non-dimensional wing half-span $(\eta)$ section of $13.06 \%$ lies outside of the immediate influence region of the bubble. An improved prediction through the chimera grid is not detectable here, see figure 9 . Both pressure and skin-friction coefficients on the upper wing surface can not be clearly discerned from each other.

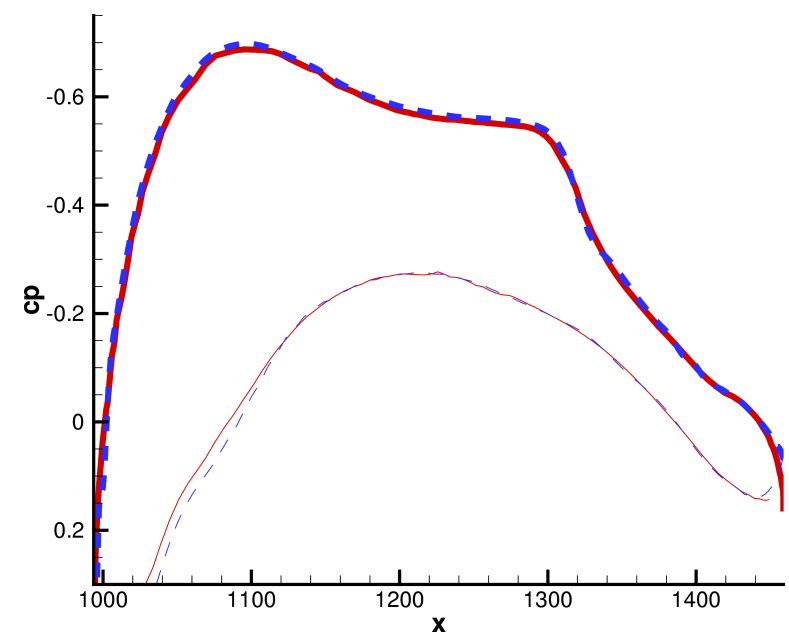

(a) Pressure coefficient (cp).

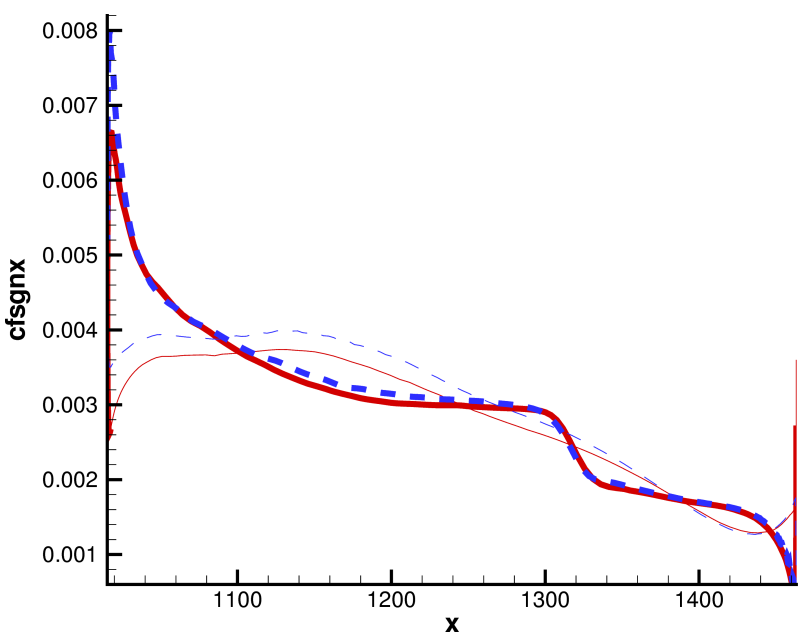

(b) Skin-friction coefficient, multiplied by the sign of the $\mathrm{x}-$ component of skin-friction (cfsgnx).

Figure 9. Case $1.1 \mathrm{Cl}=0.5$, wing cut at $\eta=13.06 \%$; bold curves for upper wing surface; Solar ( - ) and SolarChimera5 (- - ) Spalart-Allmaras results.

The innermost DPW4-required CFD data extraction cut-plane at $\eta=10.5 \%$ lies between the junction and the separation bubble eye. As visible in figure 10(b) the separation at the trailing edge is clearly seen in the 
skin-friction coefficient for the SolarChimera5 grid. Furthermore, the comparison in terms of skin-friction coefficient, shows an insufficient resolution starting from the leading edge.

In terms of pressure coefficient, see figure 10(a), the difference between the results on the two grids is not as pronounced. Even after the shock, towards the trailing edge, a strinking difference between the two results is not found. Note that due to the small, three-dimensional extent of the separated region, a clear sign of separation is not noticeable through pressure data alone.

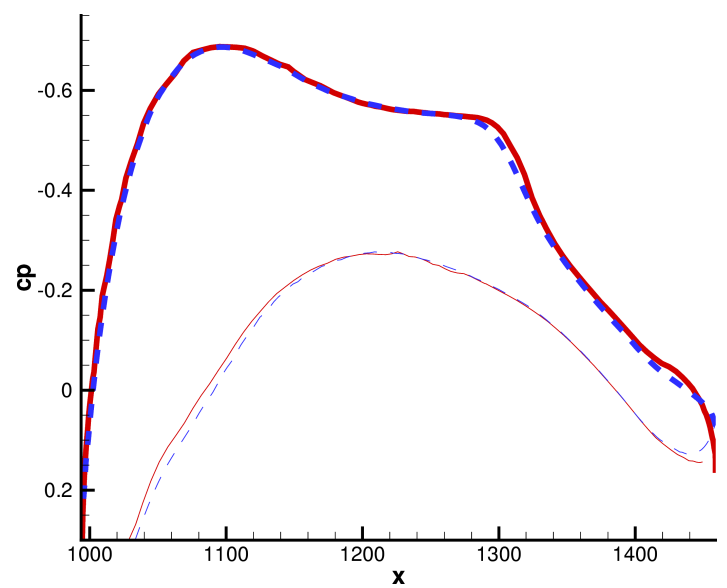

(a) Pressure coefficient (cp).

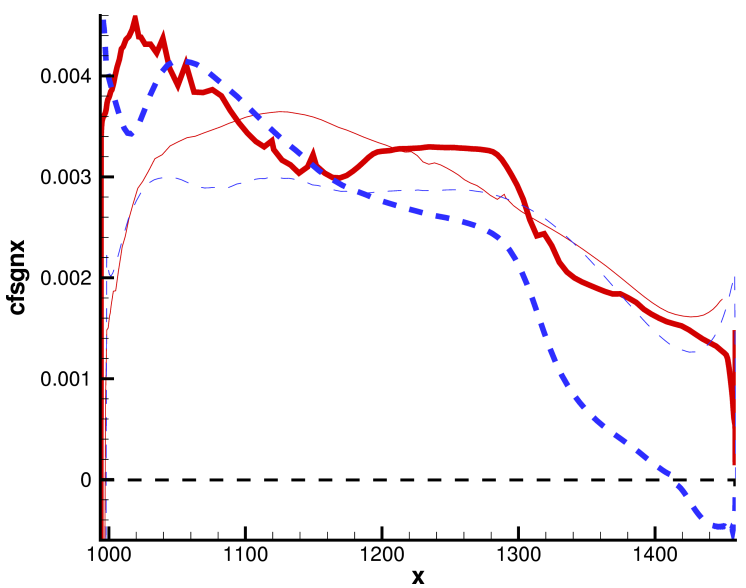

(b) Skin-friction coefficient, multiplied by the sign of the $\mathrm{x}$-component of skin-friction ( $\operatorname{cfsgnx})$.

Figure 10. Case $1.1 \mathrm{Cl}=0.5$, wing cut at $\eta=10.5 \%$; bold curves for upper wing surface; Solar ( -$)$ and SolarChimera5 $(---)$ Spalart-Allmaras results.

More advanced physical models do not improve the situation on the Solar grid. The comparison between Spalart-Allmaras (SA), Menter SST and Reynolds stress model (RSM) in figure11(a), shows virtually no difference in pressure coefficient. The levels of skin-friction coefficient (figure 11(b)) are slightly different, but the overall trend is the same; similar behavior ahead of the shock, post-shock drop-off to comparable levels and no separation at the trailing edge.

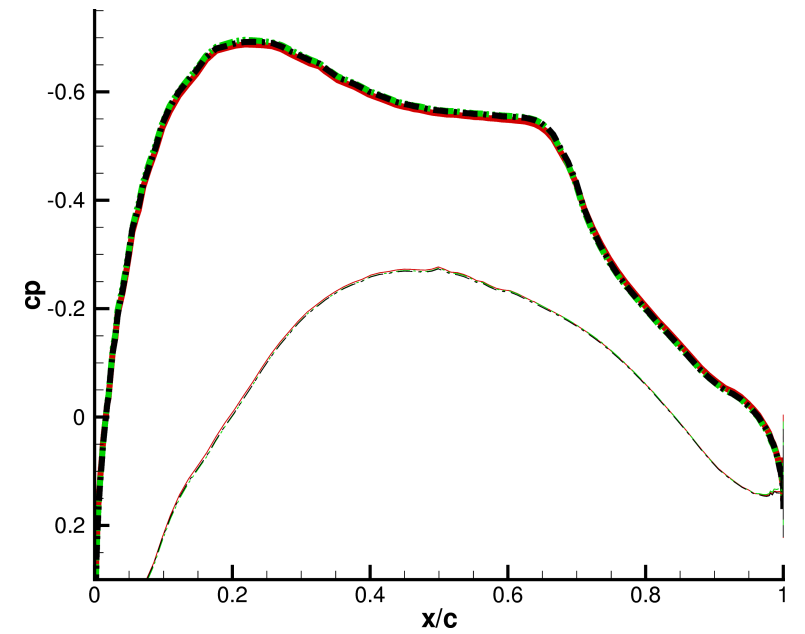

(a) Pressure coefficient.

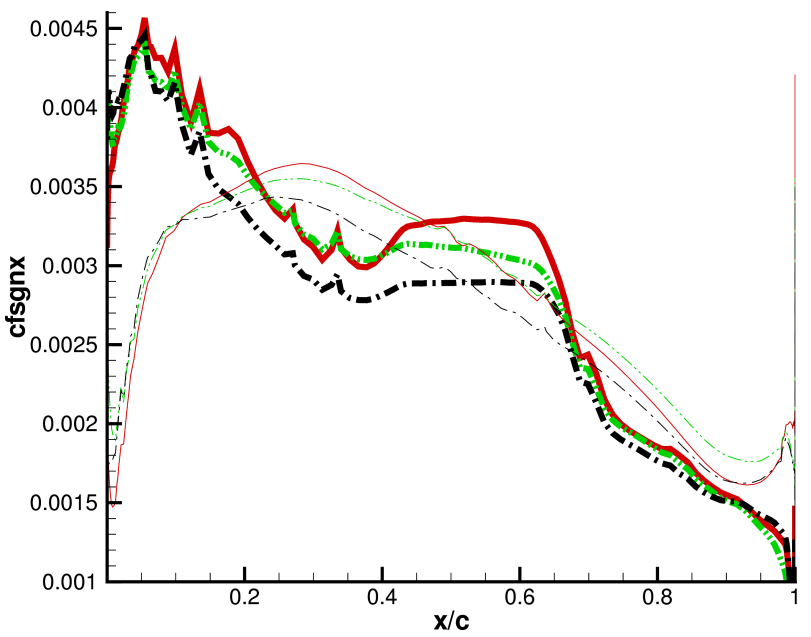

(b) Skin-friction coefficient.

Figure 11. Case $1.1 \mathrm{Cl}=0.5$, wing cut at $\eta=10.5 \%$; bold curves for upper wing surface; SA (-), SST (- - - ) and RSM $(-,-)$ results on Solar grid.

A similar comparison on the SolarChimera5 grid shows also a negligible dependence on turbulence modeling. The comparison depicted in figure 12 shows that the chosen turbulence models behave in a similar way. The difference in skin-friction at the leading edge between Spalart-Allmaras and RSM solution does not affect the region around the shock wave. The RSM result shows a more pronounced separation, due to a stronger effect of the shock wave on the boundary layer, but the separation bubble is topologically similar 
to the Spalart-Allmaras solution.

The inclusion of SARC is due to a conjectured influence on the trailing edge separation of the horse-shoe vortex emanating from the junction of wing leading edge and fairing. An effect of the rotational corrections is not noticeable on the separation bubble.

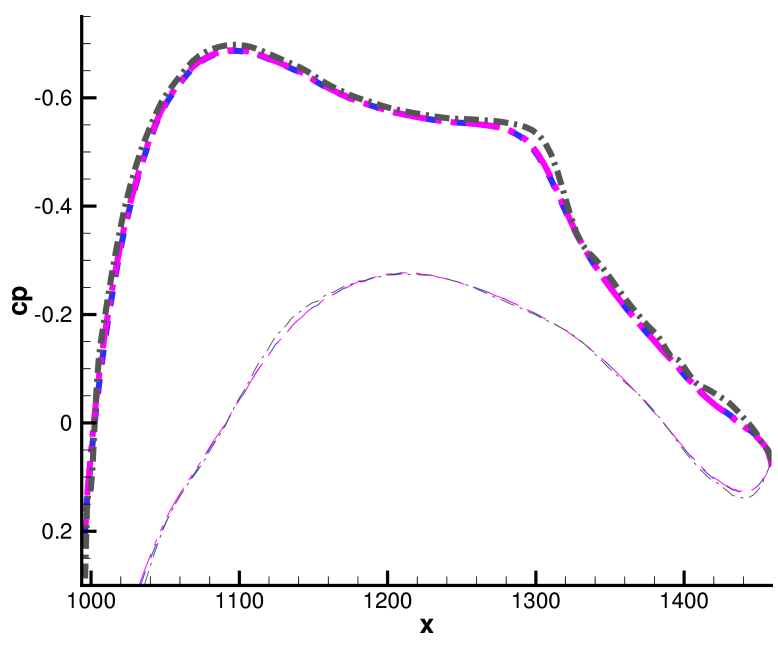

(a) Pressure coefficient.

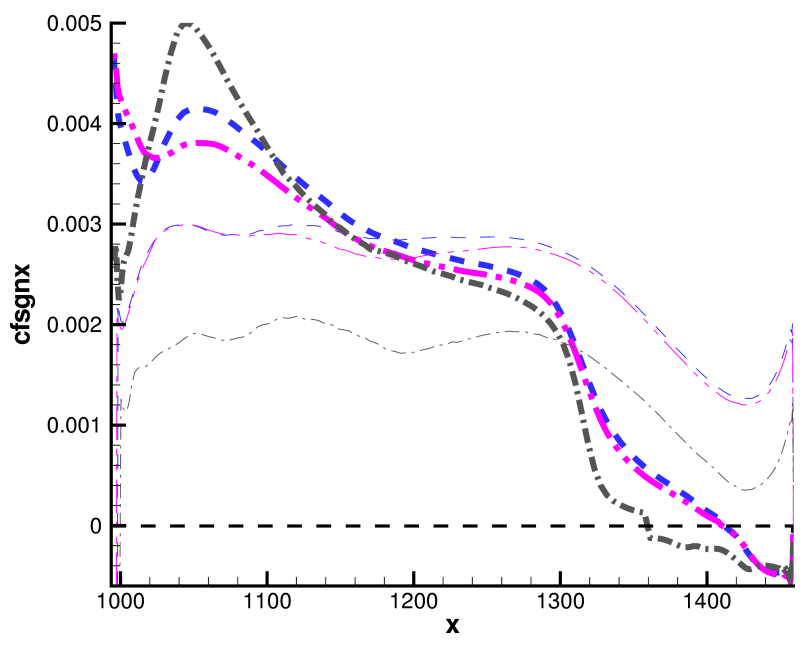

(b) Skin-friction coefficient, multiplied by the sign of the $\mathrm{x}-$ component of skin-friction (cfsgnx).

Figure 12. Case $1.1 \mathrm{Cl}=0.5$, wing cut at $\eta=10.5 \%$; bold curves for upper wing surface; SA (- - -), SARC (- . -) and RSM $(-\cdot)$ results on SolarChimera5 grid.

\section{IV.B. Case 1.2 - Edge of the Envelope}

A further comparison between the standard Solar grid and the improved SolarChimera5 grid is performed at an off-design condition. At angles of attack over $3^{\circ}$, the spread in terms of drag and moment coefficients between the contributed data increases dramatically. ${ }^{10}$ This is partly due to a major difference between the contributions in respect to wing-body separation. On the Solar grid, at $4^{\circ}$ no separation is found regardless of turbulence modeling approach (SA, SST and RSM). Comparing the DPW4-delivered dataset to the SolarChimera5 result as shown in figure 13, reveals a major influence of discretization on the wingbody junction flow field. The separation that at the target lift coefficient of 0.5 is seemingly confined at the

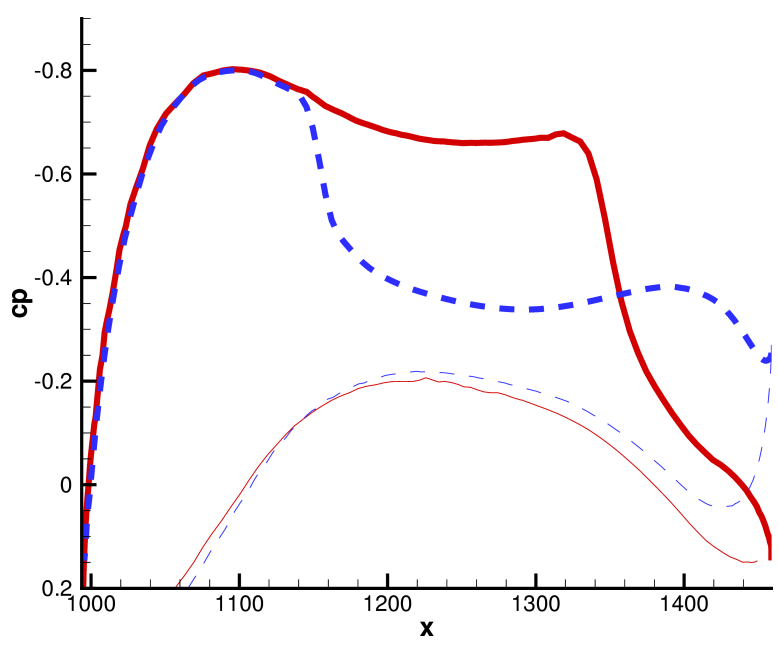

(a) Pressure coefficient (cp).

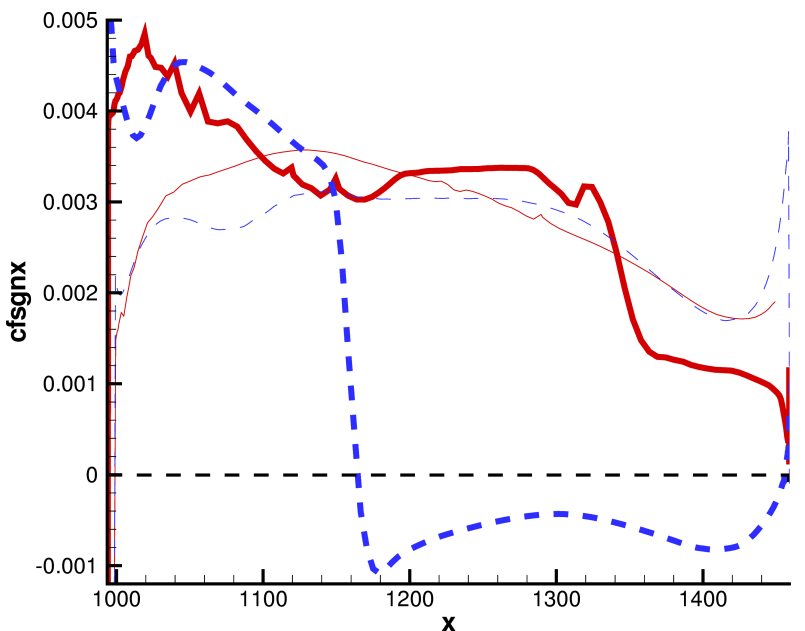

(b) Skin-friction coefficient, multiplied by the sign of the $\mathrm{x}-$ component of skin-friction (cfsgnx).

Figure 13. Case $1.2 \alpha=4^{\circ}$, wing cut at $\eta=10.5 \%$; bold curves for upper wing surface; Solar (一) and SolarChimera5 $(---)$ Spalart-Allmaras results.

trailing edge, moves upstream and starts at $x / c_{\text {root }} \approx 0.35$. As visible in figure 14 , the flow-field from the 
root to the wing kink is dominated by a substantial separation.

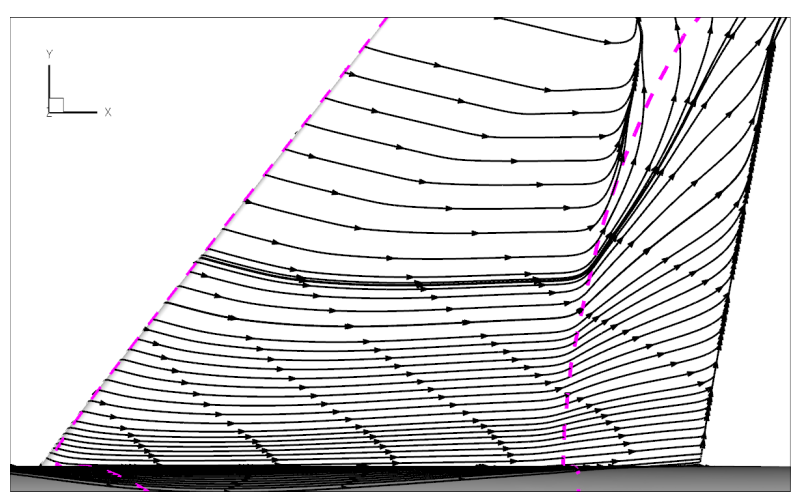

(a) Solar

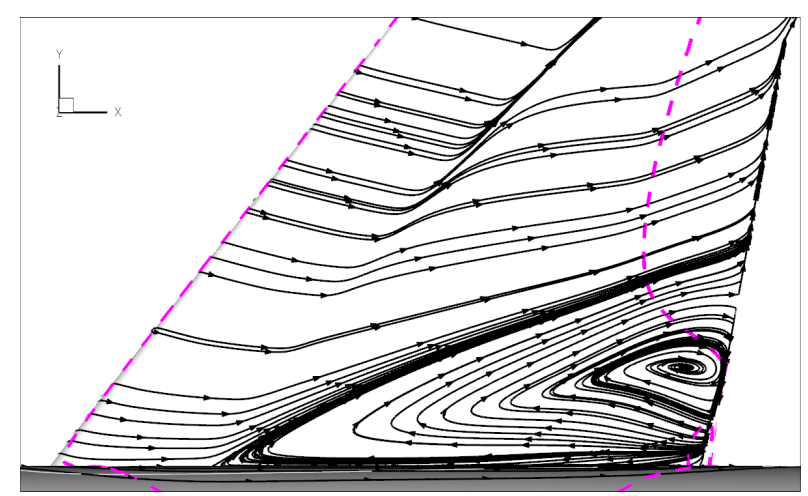

(b) SolarChimera5

Figure 14. Case $1.2 \alpha=4^{\circ}$, top view of skin-friction lines on inner wing and $c_{p, c r i t}(---)$; Spalart-Allmaras result.

\section{Conclusions}

This study documents a large amount of the grid generation efforts undertaken at DLR with the Solar meshing system for the fourth drag prediction workshop. The procedure described here to produce a grid family as self-similar as possible, was found to dramatically improve the grid convergence behavior for unstructured solutions. It remains to be seen if the approach proves successful also in future grid convergence studies, but within the context of DPW4, the grid convergence trends and levels compared to other DPW4 contributions are satisfactory. This is the case, even if the small separation bubbles at the wing and tailplane junctions with the body are not resolved. Since the separation bubbles are not resolved in any of the grid levels, the grid convergence is not affected. Fortunately the influence of the separation bubbles on drag and moment coefficients is minor at the target lift coefficient of 0.5 .

The adjoint-based dissipation error evaluations are shown to improve the hybrid CFD process accuracy through modification of the grids. The documented procedure can already support the interactive mesh generation process. The manually performed process documented here is in the process of being automated, at first through mesh adaptation, and possibly later through direct mesh generation steering.

As shown here on the "Solar" grid, with the standard mesh generation procedure it is not possible to resolve a main flow feature at the wing-body junction. For this case, an alternative solution strategy based on an overset grid ("SolarChimera5") is tested and found to improve the aerodynamic evaluation. The resolution of the separation bubble at the wing root trailing edge of the SolarChimera5 grid, is achieved without changing the solver settings. Thus the absent separation on the "Solar" grid is only due to insufficient discretization.

An analysis of the solutions on the two grids gives some insight into the flow phenomenon at the wingbody junction. The pressure rise at mid-chord of the cut-plane $\eta=10.5 \%$, is very similar between Solar and SolarChimera5 solutions, thus the strength of the shock wave is predicted similarly. It seems thus that solely the difference in boundary layer resolution is the reason for the difference between the two grids. The shock-boundary layer interaction is responsible for the initial destabilizing effect on the corner boundary layer. Only on the SolarChimera5 grid, this interaction is shown to initialize the separation, which can subsequently blow up at the trailing edge.

A sufficient discretization is not only important aft of the shock to resolve this phenomenon, but also ahead of the shock. A correct resolution of the corner boundary layer between leading edge and shock is important to capture the boundary layer development and the proper interaction with the shock wave. As shown above (figure 10(b)), the skin-friction is substantially different already ahead of the shock.

More advanced physical models are not able to capture the wing-body separation on the Solar grid, as the corner flow discretization is plainly insufficient. Only when the discretization is sufficient, one can draw correct conclusions to classify and categorize the different physical modeling approaches. 
The improvement of the corner flow discretization via the additional hexahedral mesh block, shows a major impact on the flow-field at off-design conditions. This is indeed of particular importance here, as not only the absolute values can be misleading. The trends of pitching moment and drag are potentially of more importance. The same phenomenon elucidated here on the wing-body junction is found also at the tailplane-body junction, ${ }^{9}$ which has even more profound effects on the pitching moment.

\section{References}

${ }^{1}$ Vassberg, J. C., Dehaan, M. A., Rivers, M. S., and Wahls, R. A., "Development of a Common Research Model for Applied CFD Validation Studies," 26th AIAA Applied Aerodynamics Conference, August 2008, AIAA 2008-6919.

${ }^{2}$ Schwamborn, D., Gerhold, T., and Heinrich, R., "The DLR TAU-Code: Recent Applications in Research and Industry," In Proceedings of the European Conference on Computational Fluid Dynamics, ECCOMAS CFD 2006, edited by P. Wesseling, E. Oñate, and J. Périaux, The Netherlands, 2006.

${ }^{3}$ Spalart, P. R. and Allmaras, S. R., "A One-Equation Turbulence Model for Aerodynamic Flows," La Recherche Aérospatiale, Vol. 1, 1994, pp. 5-21.

${ }^{4}$ Menter, F. R., "Two-Equation Eddy-Viscosity Turbulence Models for Engineering Applications," AIAA Journal, Vol. 32, 1994, pp. 269-289.

${ }^{5}$ Eisfeld, B., "Numerical Simulation of Aerodynamic Problems with the SSG/LRR- $\omega$ Reynolds Stress Turbulence Model Using the Unstructured TAU Code," New Results in Numerical and Experimental Fluid Mechanics VI, Vol. 96 of Notes on Numerical Fluid Mechanics and Multidisciplinary Design, Springer-Verlag, 2007, pp. 356-363, 15. DGLR/STAB-Symposium.

${ }^{6}$ Leatham, M., Stokes, S., Shaw, J. A., Cooper, J., Appa, J., and Blaylock, T., "Automatic Mesh Generation for RapidResponse Navier-Stokes Calculations," FLUIDS 2000 Conference and Exhibit, June 2000, AIAA 2000-2247.

${ }^{7}$ Brodersen, O., Crippa, S., Eisfeld, B., Keye, S., and Geisbauer, S., "DLR Results from the Fourth AIAA CFD Drag Prediction Workshop," 28th AIAA Applied Aerodynamics Conference, June 2010, AIAA 2010-4223.

${ }^{8}$ Dwight, R. P., "Heuristic a posteriori estimation of error due to dissipation in finite volume schemes and application to mesh adaptation," J. Comput. Phys., Vol. 227, No. 5, 2008, pp. 2845-2863.

${ }^{9}$ Schwarz, T., "An Interpolation Method Maintaining the Wall Distance for Structured and Unstructured Overset Grids," Proceedings of the CEAS 2009 conference, CEAS 2009 European Air and Space Conference, October 2009.

${ }^{10}$ Vassberg, J. C., Tinoco, E. N., Mani, M., Zickuhr, T., Levy, D. W., Brodersen, O., Crippa, S., Wahlsk, R. A., Morrison, J. H., Mavriplis, D. J., and Murayama, M., "Summary of the Fourth AIAA CFD Drag Prediction Workshop," 28th AIAA Applied Aerodynamics Conference, June 2010, AIAA 2010-4547. 